\title{
Substation area joint defensive protection strategy based on distributed cooperative all-in-one device
}

\author{
Fanrong WEI ${ }^{1}$, Mengqi YU ${ }^{1}$, Zhiqian $\mathrm{BO}^{2}$, Kun $\mathrm{YU}^{1}$, \\ Jingguang HUANG ${ }^{3}$
}

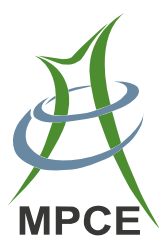

\begin{abstract}
Integration of primary and secondary devices are impelled by the development of smart grid. Based on the ideology of primary and secondary device integration, an all-in-one device and its concept, configuration and function are proposed. Then, an all-in-one device framework of distributed substation area protection is proposed. Point-topoint model and virtual local area network (VLAN) based network-to-network model are adopted in this framework. The dual redundancy on hardware and software level is realized by modularization and protection information mirroring storage/overwriting. Considering the fact that the sampling, logic judgment and information sharing processes of protection may fail in atrocious conditions, a distributed cooperative all-in-one device cluster based substation area joint defensive protection strategy is
\end{abstract}

CrossCheck date: 30 May 2016

Received: 30 November 2015/Accepted: 30 May 2016/Published online: 12 July 2016

(C) The Author(s) 2016. This article is published with open access at Springerlink.com

$\triangle$ Fanrong WEI

weifanrong@gmail.com

Mengqi YU

woyumengqi@gmail.com

Zhiqian BO

bozhiqian@263.net

Kun YU

1393009168@qq.com

Jingguang HUANG

258926016@qq.com

1 Huazhong University of Science and Technology, Wuhan, China

2 XUJI Group, Beijing, China

3 Three Gorges University, Yichang, China proposed. The adaptability of sectional component failure in secondary system is strengthened by reusing module function, setting value mirroring storage and dynamic load balancing computing ability to constitute strong intelligent outdoor secondary device integrated network. Finally, the simulation examples based on EPOCHS which consider various system failure conditions are presented to verify the validity and rationality of the proposed architecture and strategy.

Keywords All-in-one device, Substation area protection, Distributed cooperation, Protection overwrite, Joint defensive protection

\section{Introduction}

Integration of primary and secondary device is an important development direction of smart grid in the future $[1,2]$. By packing the sensing measurement, logic computing and communication cooperation ability of secondary intelligent unit into primary devices with the support of fiber, self-organizing substation interactive protection and control network system can be realized [3]. According to the principle of modular design, the traditional substation secondary system construction process can be simplified and the assembly errors that may occur in the construction process can be remarkably reduced [4], which gives the substation integrated solution an incomparable advantage to reshape the existing market of equipment manufacturing.

If the previous scheme designs of substation area protection and wide area protection are adopted directly to address the need, there will be lots of problems in suitability and reliability. Mounts of previous works focused on substation protection. A novel reliability modeling and 
analysis methodology were proposed in [5] for modern substation protection systems. An integrated control and protection system are proposed in [6] with a hierarchical coordination control strategy. In [7], a substation backup protection scheme based on multiple limitedly overlapped divisions was proposed. In general, the integrated protection or the substation area protection usually acts as backup protection of substation, especially in high voltage systems. However, the reliability of this type of structure is still in doubt, related technologies and strategies cannot directly apply to such a design that integrates the primary equipment and secondary device into an entity. Although seldom adopted in substation area protection, the distributed protection mode is widely accepted in the field of wide area protection. In [8], a study of communication was presented based on wide area agents for primary and backup coordinated protection. A multi-agent based adaptive wide area current differential protection system was proposed in [9]. In [10], a faulted element identification algorithm was proposed based on multisource information fusion for wide area protection. A novel wide area backup protection algorithm was presented in [11] using information shared across wide area communication networks. In [12], a multi-agent system based wide area protection and control scheme was proposed to deal with the long-term voltage instability induced cascading trips. The researches on distributed protection are valuable for the integration of primary or secondary devices and for that an agent or a distributed unit works in the same way in the wide area or the substation area. However, the intelligent secondary devices designed to be placed in substation master control room cannot well match the life span of primary devices. Besides, their adaptation for strong electromagnetic interference and atrocious operation environment is yet to be testified [13]. Therefore, there are lots of problems in feasibility and reliability of the integration of primary device and secondary device in the same equipment.

Accounting for the running environment of primary and secondary device integration, the conception of all-in-one device and a distributed centralized protection structure are proposed to deal with those problems from the aspects of the topology, hardware and software design. The secondary devices originally planned to be installed in the primary device are now separated into different functional secondary device clusters into equipment cabinets with favorable environment according to their geographical locations and are designed based on modular structure. Its configuration and function are described in detail. Then, the virtual local area network (VLAN) networking scheme based on secondary all-in-one device cluster is proposed. To solve the problem of reliability, protection unit failure is dealt with using adjacent back-up protection which will extend trip range [14-16], all-in-one device based substation area joint defensive protection strategy is proposed in this paper. Moreover, the general-purpose computing (GPC) module protection switching mechanism is analyzed. The adaptability of sectional component failure in secondary system is strengthened by reusing module function. The proposed joint defensive protection strategy is particularly analyzed in various fault conditions. Finally, the feasibility and advantage of the protection defense strategy with the support of high speed Ethernet are testified by EPOCHS based joint simulation example.

\section{All-in-one device}

The internal structure of the all-in-one device is shown in Fig. 1. Each device is equipped with one CPU and several GPCs, CPU is responsible for task scheduling and monitoring while the GPCs act just the same as co-processors. The device can collect voltage and current measurement in the primary side as well as switch signals, such as the position nodes of breakers and disconnectors. It can make full use of its own processor to realize the fast protection in the form of local measuring and local tripping. Besides, the all-in-one device can provide comprehensive information on this side of the cross-bay protection in the substation. One or a plurality of all-in-one devices can quickly make up the cross-bay protection using communication network within the station, such as transformer differential protection, bus differential protection and so on.

Compared with the traditional devices, the proposed device is designed with hardware self-redundancy and communicating module. Moreover, the setting value of the traditional protection is only stored by the device itself, without setting-value mirroring storage which managed by system. However, this function can be fulfilled in the proposed designed. The all-in-one device conforms to the advantage of such design as integrating primary and secondary devices into an entity. Then, the compact structure of local layer can be realized. Meanwhile, the function of the bay level is still retained. And cross-bay protection based on all-in-one device does not require concrete protection devices, which further reduces the occupied area of

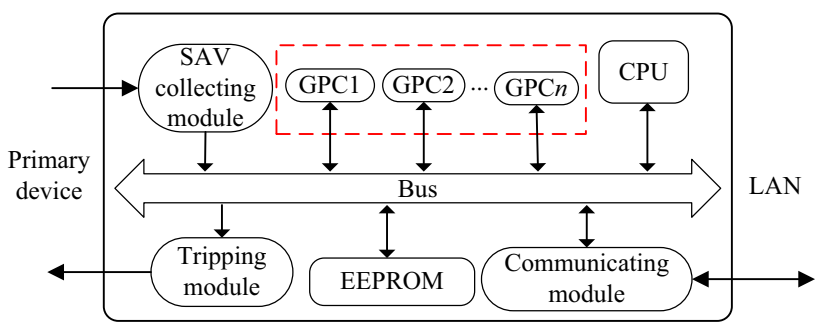

Fig. 1 Inner structure of all-in-one device 
the secondary equipment. The proposed topological structure based on all-in-one device applies equally to primary and secondary device integration. Meanwhile, it avoids the adaptation for strong electromagnetic interference and atrocious operation environment to some degree.

\subsection{SAV collecting module}

All-in-one device can collect real-time data, mainly including voltage, current and breaker status from other sampling devices and preprocess the collected data through general protection principle. Then it organizes the data in a certain format and then sends the frame to the data bus of the all-in-one device.

\subsection{Communicating module}

By the communication network, all-in-one device shares the area fault information and receives the tripping decision to cut off the fault. All-in-one device needs the communication capacity of high margin, high communication speed and powerful information processing capability to support hierarchical processing and real-time throughput for the huge amount of information from both inside and outside the station. It can provide quick and reliable responses and cooperate with other devices.

\subsection{GPC module}

GPC is an independent plug-in module which can read the necessary electrical information, according to the mapping relationship of the program, within Ethernet to identify faults. All-in-one device obtains fault information of protection area and identifies the fault with the integrated decision recognition algorithm. When the identification result is uncertain, it starts a new round of negotiation and takes fault-tolerant measures to identify faulty components.

\subsection{Setting-value mirroring storage}

The SCD file format of the substation's protection setting-value mirror is shown in Fig. 2. The describing part of the protection IED includes two parts. The first part is the protection setting-value information of the original device. The other part is the setting-value overwriting information which comes into operation in the lack of information when fault occurs. In the latter fault scenario, SCD analysis module is responsible for extracting the setting-value description information of the replaced protection unit at the distal end and the pre-designed protection logic algorithm to deal with different faults from files. Through building a proprietary protection overwriting virtual

\begin{tabular}{|c|c|c|c|}
\hline Head message & $\begin{array}{c}\text { Substation } \\
\text { message }\end{array}$ & Protection 1 IED & $\begin{array}{c}\text { Communication } \\
\text { description }\end{array}$ \\
\hline Head message & $\begin{array}{c}\text { Substation } \\
\text { message }\end{array}$ & Protection 2 IED & $\begin{array}{c}\text { Communication } \\
\text { description }\end{array}$ \\
\hline Head message & $\begin{array}{c}\text { Substation } \\
\text { message }\end{array}$ & Protection 3 IED & $\begin{array}{c}\text { Communication } \\
\text { description }\end{array}$ \\
\hline$\ldots$ & $\ldots$ & $\ldots$ & $\ldots$ \\
\hline Head message & $\begin{array}{c}\text { Substation } \\
\text { message }\end{array}$ & Protection $n$ IED & $\begin{array}{c}\text { Communication } \\
\text { description }\end{array}$ \\
\hline
\end{tabular}

Fig. 2 Substation protection unit setting-value mirror SCD file format

network, the information can be imported into the local allin-one device to realize alternative protection and improve the reliability of the station protection.

\section{Distributed cooperative substation area protection system}

\subsection{System structure}

The structure of distributed cooperative substation area protection system is shown in Fig. 3. Based on all-in-one devices, the system structure divides the substation into several parts. Some bays are supervised by a single device so that the total number of secondary devices can be reduced. The protection and control functions are achieved directly by the distributed installed all-in-one devices, thus forming a protection system based on the all-in-one device in the substation layer. Distributed devices cluster improve the reliability of protection, the cancellation of main control room reduce the land occupied.

Considering that the substation is generally operating under bus splitting mode, and single all-in-one device can centrally supervise more than one bay. The area (or number of bays) to be supervised by a single all-in-one device should be determined following the criteria below.

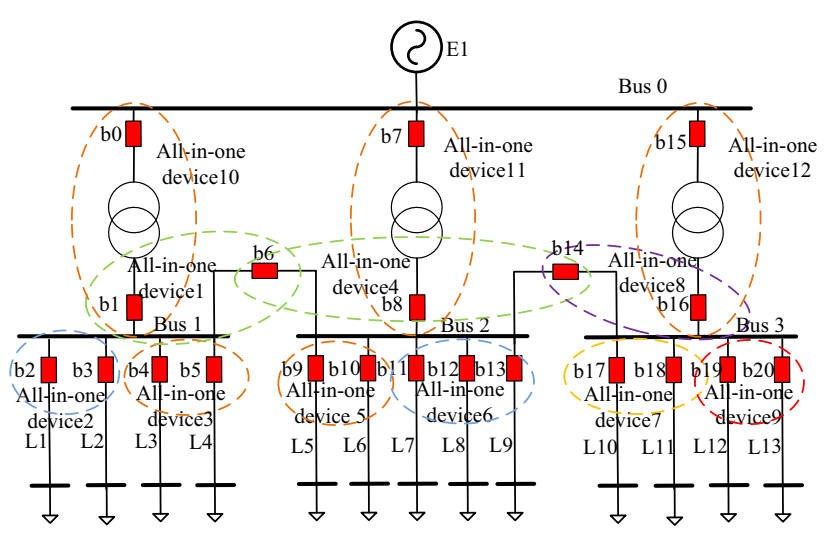

Fig. 3 Joint defensive structure of substation area protection 
1) The computing ability and hardware scale of all-in-one devices.

2) The bays geographically being close should be supervised by same device.

3) The arrangement of the main and back-up protection in a single bay.

4) The reliability of all-in-one device.

All-in-one device can not only realize the fast protection of single bay, but also provide comprehensive information on this side of the cross-bay protection in the substation. Taking the hardware redundancy into account, 6-10 modules are required for each bay. At this stage, the number of small plug-in type industrial control equipment can be compatible with 2-4 bays. However, it is only an approximate estimate based on the existing integrated degree of hardware and the reliability of device. Along with the progress of electronic technology and communication technology, the area to be supervised by a single allin-one device will further be improved.

Thus it needs a plurality of all-in-one devices to work together in order to constitute the bus protection, as shown in Fig. 3. The all-in-one device1 is in charge of the lowvoltage side of the transformer. The all-in-one device 2 is in charge of the outlet lines L1 and L2. The all-in-one device3 is in charge of the outlet lines L4 and L5. The protection of bus 1 is cooperatively formed by device $1,2,3$. Generally, the bays geographically close are supervised by a same device.

\subsection{VLAN network scheme}

The achievement of the function of distributed cooperative substation area protection system depends on a strong communication framework, which ensures that the information can be quickly and correctly transferred. Thus the communication requirements can be met by constructing a VLAN. VLAN is built on LAN switches, it divides LAN equipment from the logical partition (not physically) into several segments (smaller LAN), to realize data communication of internal virtual work group. The network formed by VLAN can shield the public network structure and encrypt the data, which turns the whole network into an internal network on logic. The VLAN can offer an economy, safe, flexible and comfortable information communication solution for all-in-one devices.

By combining the scope partition of the substation protection, the VLAN networking scheme within the substation can be formed, as shown in Fig. 4. The VLAN partition mode based on port is adopted. According to the actual demand, a port can be divided into different VLAN. The all-in-one devices constituting the same sectionalized bus protection can communicate rapidly via the VLAN,

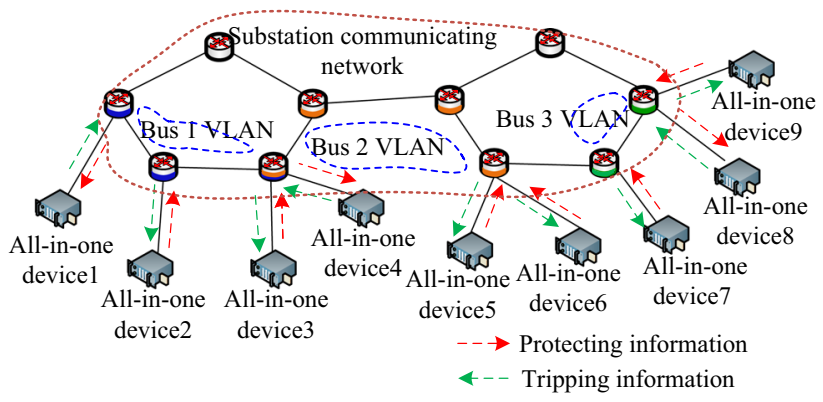

Fig. 4 VLAN of distribution centralized substation

thus achieving the isolation from the information of other sectionalized buses. The all-in-one devices of different sectionalized buses can transmit information through the distributed substation area communication network.

\subsection{Overwriting mechanism of faulty GPC}

An all-in-one device generally equips with several GPCs, as seen in Fig. 5. When all protection logic algorithm need to be realized, part of the GPCs are at work (solid line), and the others act as backup (dotted line).

Assuming that there is a GPC failure (shading), it needs to call the mirror to overwrite the backup GPC according to the following steps:

1) Locate the backup GPC by topology searching and give priority to overwriting the GPC of the same device, thus achieving the switchover within the device. This is shown by message1 in Fig. 5. When all the backup GPCs of the same device have been in use, the process of overwriting the GPCs of the

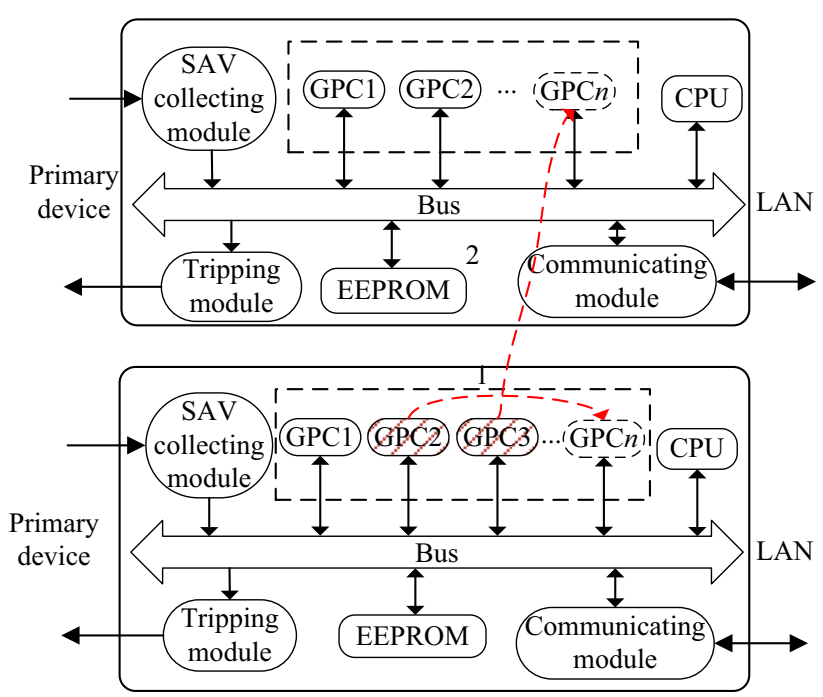

Fig. 5 Overwriting mechanism of faulty GPC 
adjacent device is inevitable. This is shown by message 2 in Fig. 5.

2) Locate the mirror of the fail-to-work protection module, which behaves as a certain GPC, and overwrite this specified GPC with above located mirror.

3) Cancel the original record of the corresponding protection and broadcast in LAN, update software and hardware list of existing protection and acquire I/O permissions.

After the steps above, the transfer of protection software between different hardware can be achieved. Essentially, another VLAN network has been added after the physical address of the protection is changed. Therefore, VLAN domain is required to be redefined in accordance with the changes in the communication topology.

\subsection{Overwriting mechanism of sampling value loss}

Unlike GPC, an all-in-one device equips with one SAV collecting module. Therefore, it's necessary to design a novel overwriting mechanism when sampling value is missing. Supposing that there is an all-in-one device failure with sampling value loss, the overwriting mechanism is different when the device is in different topologies. On the one hand, if the adjacent devices can constitute a closed loop, it will overwrite a differential protection algorithm to the adjacent devices through the private protection overwritten virtual network; on the other hand, if the adjacent devices cannot constitute a closed loop, it will degrade the protection of the superior devices. For instance, replace the original differential protection by the overcurrent protection as the bus protection.

\section{Joint defensive protection strategy}

\subsection{Description of distributed cooperative groups}

The all-in-one device can obtain and process a large number of concurrent information comprehensively in real time and are endowed with communication channel with other devices via VLAN network in the substation. If one protection device fails in harsh outside environment in all aspects of sampling, logic judgment, communication, the surviving device can form cooperative group and temporarily replaced the work of faulty device initiatively by overwriting vacant calculation module from mirroring storage. With the joint defensive protection system constituted by a plurality of all-in-one devices, the flexible scheduling and normal device of the secondary system can be ensured while the reliability of which cannot be influenced by small scale indeterminate faults.

\subsection{Achievement of joint defensive protection}

\subsubsection{Normal device}

To ensure the efficiency of data transmission and avoid network congestion in emergency conditions that a torrent of SAV data pouring into network, VLAN mode is introduced to the process of system integration that all-in-one device can only share information via VLAN with the ones that involved in the same bus segment protection. For example, the Bus 1 protection is formed by device1 to device3. They share information via VLAN 1. However, Buses 1, 2, 3 are in no need to exchange information under normal circumstances.

Each device can obtain the information of the other devices via VLAN and participate in the protection calculation. To ensure the accuracy and reliability of judgment, the weighted three select two strategy is adopted. The expression $\frac{2}{3}\left(n_{1} k_{1}+n_{2} k_{2}\right)$ is the weighed sum of devices making the final judgment; $n_{1}$ is the number of devices that supervises the bus inlet line; $n_{2}$ is the number of devices that doesn't supervise the bus inlet line; $k_{1}$ and $k_{2}$ are weighted coefficients $\left(k_{1}>k_{2}\right)$ which are adopted to weight the importance of different bay; $[X]$ is the integer part of $X$. The final judgments depend on the devices computing faster, there is no need to wait for the judgments of all the device. Taking Bus 1 as example, set $n_{1}=1$, $n_{2}=2, k_{1}=2, k_{2}=1$, then $\frac{2}{3}\left(n_{1} k_{1}+n_{2} k_{2}\right)=2$, the final judgments of Bus 1 is shown in Table 1 . The proposed strategy is also applicable to other protection in substations, its description will not be repeated here.

\subsubsection{Failure of GPC}

By virtue of the configuration features of GPC, the overwriting mechanism is the same when the faulty device is in different topologies. If the GPC of all-in-one device1 fails, it can load remote mirroring protection of the faulty GPC and overwrite the backup GPC in the same device by private virtual network. Furthermore, if all the backup

Table 1 Judgments of Bus 1

\begin{tabular}{llll}
\hline $\begin{array}{l}\text { Weighed } \\
\text { device } 1\end{array}$ & $\begin{array}{l}\text { Weighed } \\
\text { device 2 }\end{array}$ & $\begin{array}{l}\text { Weighed } \\
\text { device } 3\end{array}$ & $\begin{array}{l}\text { Final } \\
\text { judgments }\end{array}$ \\
\hline 1 & & & 1 \\
1 & 1 & 1 & 1 \\
1 & 1 & 1 & 1 \\
1 & 1 & 1 & 1 \\
\hline
\end{tabular}

Note: "1" means fault 


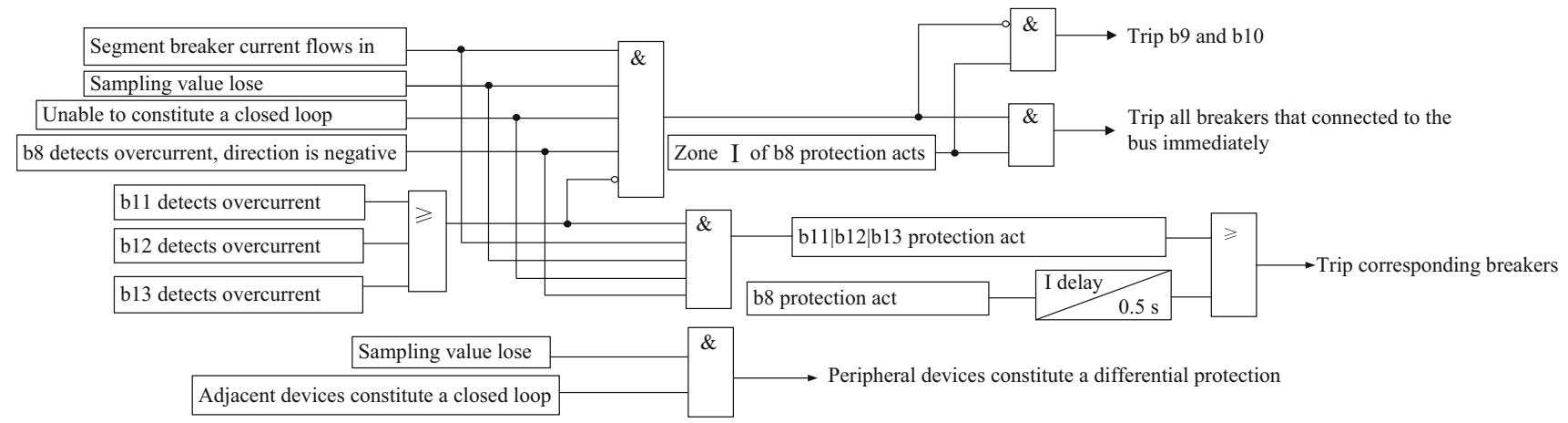

Fig. 6 Comprehensive overwriting mechanism of sampling fault

GPCs are overwritten with new protection, it needs to be replaced in the adjacent ones, such as the GPCs in all-inone devices 2 and 3 .

\subsubsection{Failure of SAV collecting module}

With regard to the configuration features of SAV collecting module, the overwriting mechanism is different when the faulty device is in different topologies.

1) If the SAV collecting module of all-in-one device5 is failed, as a result, the Bus 2 differential protection will not be able to continue to play a proper role. Meanwhile, the adjacent devices (devices 4 and 6) cannot form a closed loop without device5, as shown in Fig. 3. Under this circumstance, the strategy to

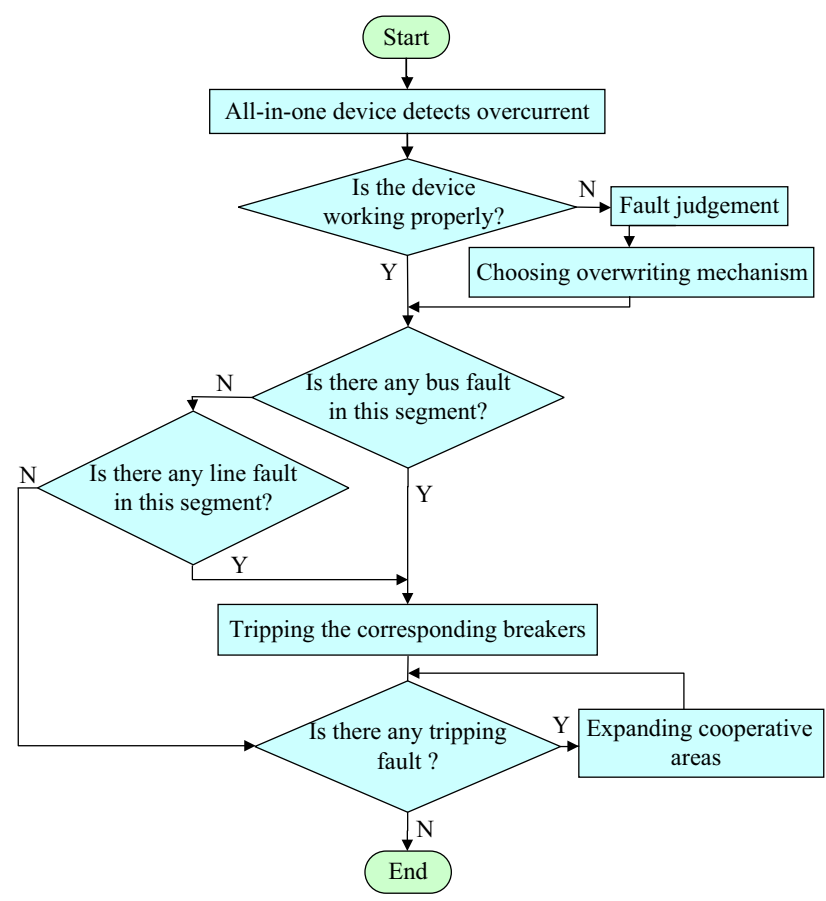

Fig. 7 Substation area joint defensive protection strategy process overwriting superior breaker b8 by VLAN network would be a proper choice. It should be noted that the more sensitive overcurrent protection of b9 and b10 is used to overwrite b8. Moreover, the cooperation of b8, b11, b12, and b13 should be synthetically considered. Firstly, the corresponding device sends a blocking message to the b8 as long as an overcurrent is detected by b11, b12, or b13, delaying $0.5 \mathrm{~s}$. Secondly, if b11, b12 or b13 did not detect an overcurrent and zone I protection of $b 8$ act, it should remove all the breakers that connected to the bus immediately; if zone I overcurrent protection of b8 did not act and zone II, III act, b9 and b10 should be disconnected.

2) If the sampling value of b8 is invalid, thus the Bus2 differential protection will be out of work. However, the adjacent breakers b6, b7, b9, b10, b11, b12, b13, b14 can still constitute a closed loop, as shown in

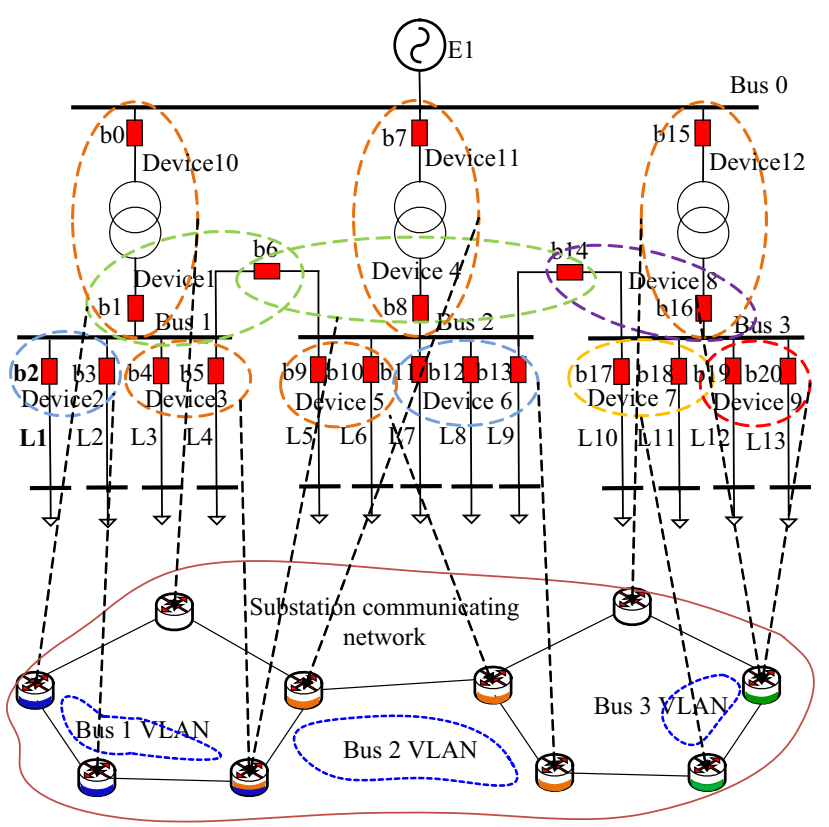

Fig. 8 Substation power system and communication structure 
Fig. 3. Therefore, it will overwrite a differential protection algorithm to the adjacent devices.

\subsubsection{Joint defensive protection strategy based on all-in- one devices}

The all-in-one devices can realize the joint defensive protection through cooperation, even though, parts of them are out of work. The secondary system is still able to quickly respond to various kinds of faults, forming a strong secondary integration network. The whole process is shown in Fig. 7.

According to Fig. 3, a comprehensive protection overwriting mechanism is proposed in order to overcome

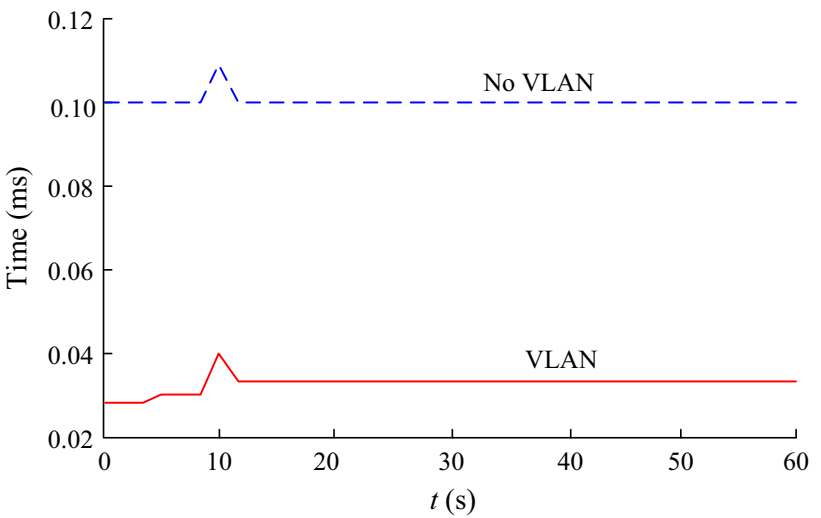

Fig. 9 Influence of VLAN on Ethernet delay

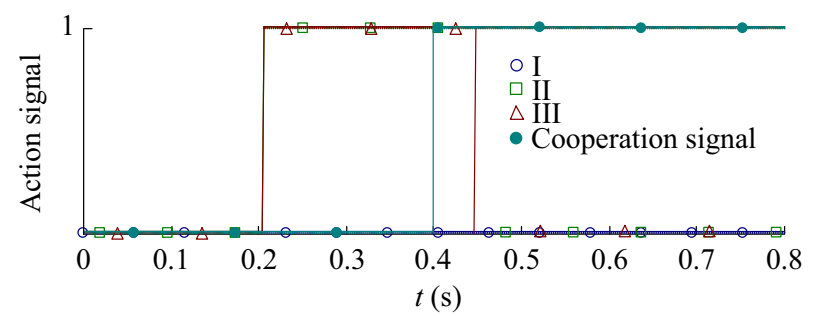

(a) b3 local measurement action signal and cooperation decision signal

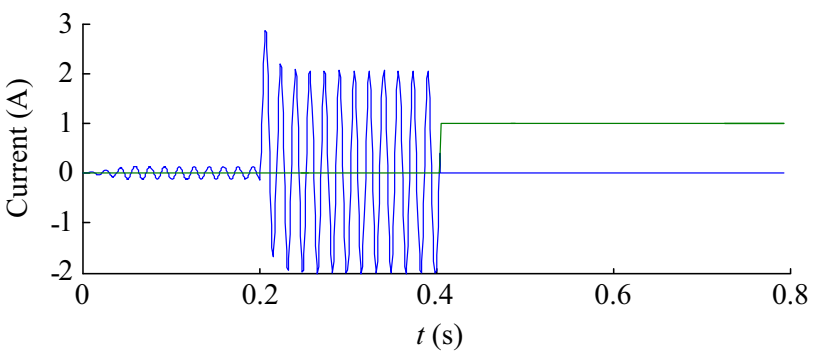

(b) b8 corresponding line current waveform

Fig. 10 Line protection under normal circumstance sampling value loss, as shown in Fig. 6. However, this kind of strategy can only be used temporarily as an emergency alternative for short-term transition.
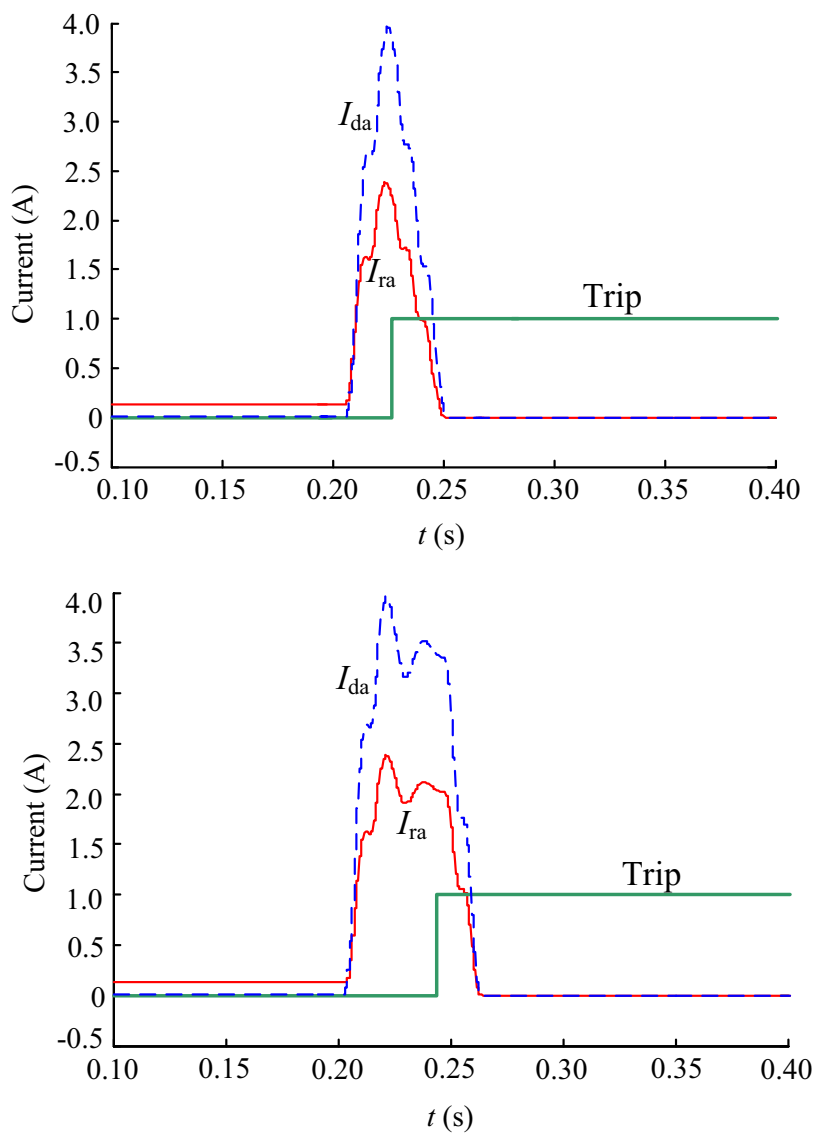

(a) Differential and restrain current of device4 and 6
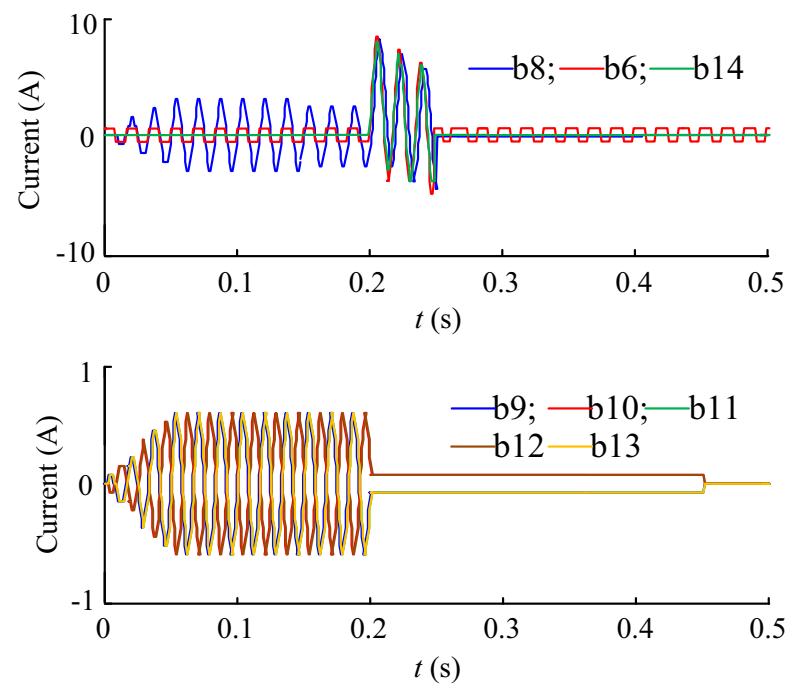

(b) Current waveform of lines connect to faulty bus

Fig. 11 Bus protection with faulty GPC 


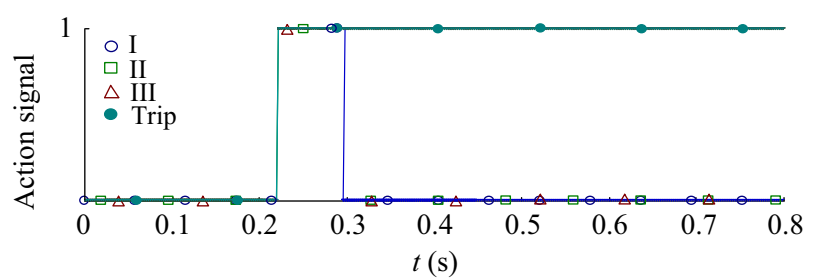

(a) b8 local measuring action signal and tripping command

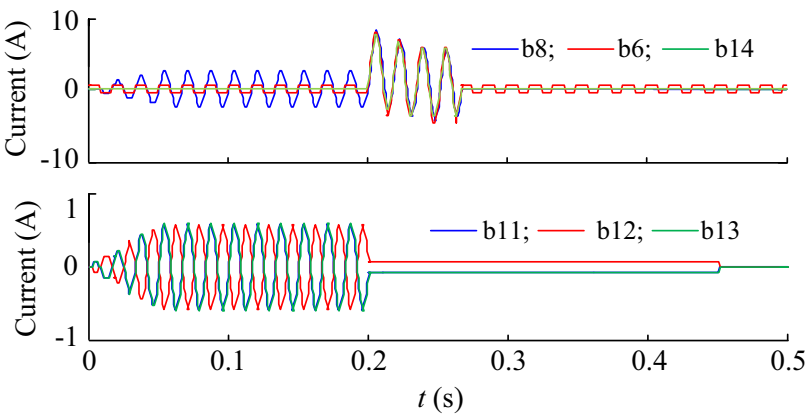

(b) Current waveform of lines connect to faulty bus

Fig. 12 Bus segment2 fault while b9 and b10 sampling value miss

\section{Simulation tests and analysis}

Based on the EPOCHS synchronous simulation platform of power grid and communication system, the system simulation model of the $110 \mathrm{kV}$ substation is established, as shown in Fig. 8. In the model, there are three 50/50 MW transformers. $10 \mathrm{kV}$ side bus uses segment connection and segment breaker is switch on. Each all-in-one device controls 2 to 4 sampling points/breakers, distributed according to the location of the primary device and accessed into $100 \mathrm{M}$ bandwidth of station communication network. The breaker action time is set to $50 \mathrm{~ms}$. By the simulation experiments on NS2, the solid line represents the end-to-end delay of the same bus segment is $0.02 \sim 0.04 \mathrm{~ms}$ via VLAN, as shown in Fig. 9, such as from device 1 to device 2 via VLAN 1 in Fig. 8. The dotted line represents the far end-to-end delay of the different bus segment is $0.1 \sim 0.12 \mathrm{~ms}$, such as from device 1 to device 5 via substation LAN in Fig. 8. The simulation results show that the VLAN can significantly reduce the network delay.

\subsection{Normal device}

Scenario 1: A ground fault occurs on the end of L2 is simulated, overcurrent protection zone I of b3 did not trip, while zone II, III did. Meanwhile, all-in-one device2 receives the message that the overcurrent protection of $b 2$, b4, b5 did not trip. Then, substation area protection is adopted to locate the fault. Correspondingly, it will

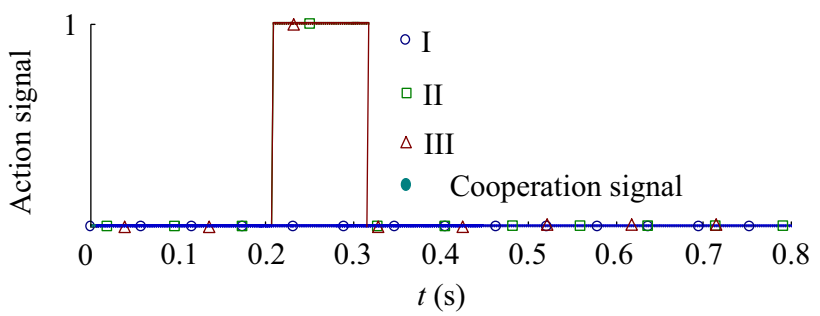

(a) b8 local measuring action signal

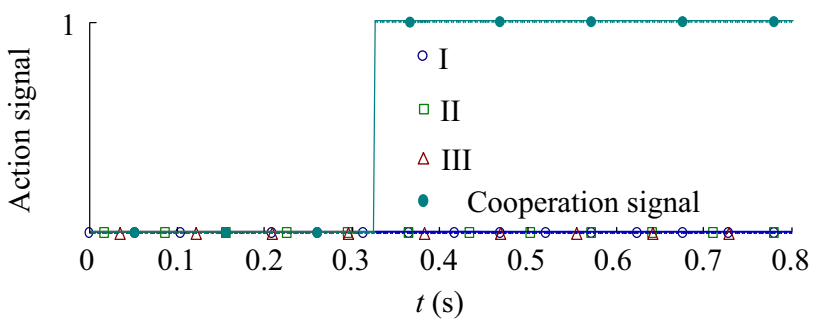

(b) b9 or b10 local measurement action signal and cooperation decision signal

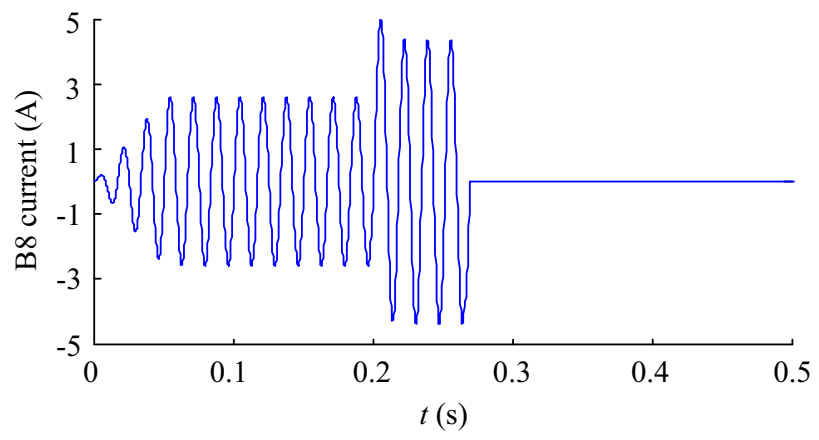

(c) b8 corresponding line current waveform

Fig. 13 L5 failure while b9 and b10 sampling value miss

accelerate the overcurrent protection zone II trips as long as the fault occurs on L2, without delaying $0.5 \mathrm{~s}$ for the zone II of traditional protection, as shown in Fig. 10.

\subsection{Failure of GPC}

Scenario 2: A ground fault occurs on Bus 2 is simulated. Meanwhile, a GPC fault occurs on all-in-one device5. The weighted three select two strategy is adopted. When a fault occurs on GPC of device5, the judgments of device 4 and 6 are shown in Fig. 11a, the current waveform of the lines connect to faulty bus is shown in Fig. 11b. The response speed of protection is similar to the sound device.

\subsection{Failure of SAV collecting module}

Assuming that the SAV collecting module of all-in-one device5 is fault. As a result, the sampling values of b9 and b10 are missing, as shown in Fig. 8 . 


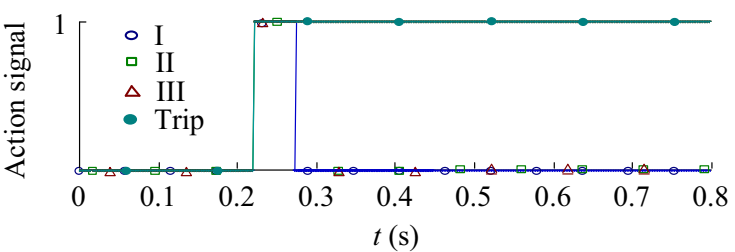

(a) b11 local measuring action signal and tripping command

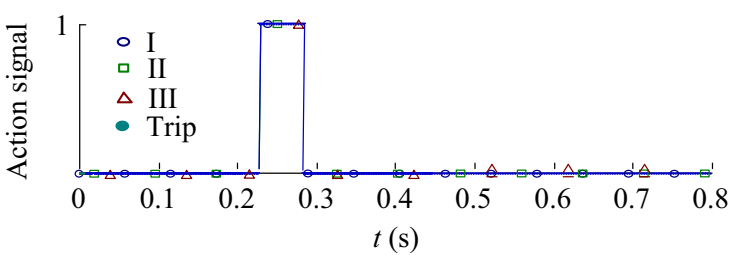

(b) b8 local measuring action signal

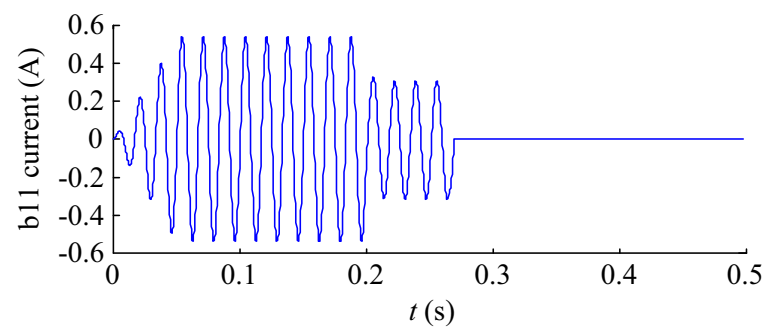

(c) b11 corresponding line current waveform

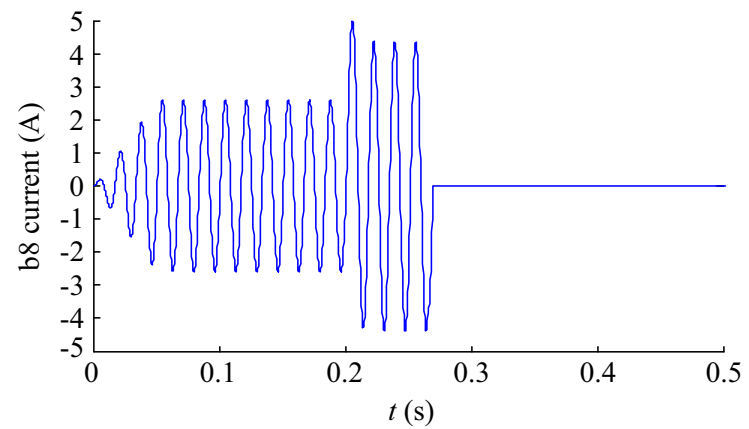

(d) b8 corresponding line current waveform

Fig. 14 L7 failure while b9 and b10 sample value miss

Scenario 3: A ground fault occurs on Bus 2 at $t=0.2 \mathrm{~s}$ is simulated. Meanwhile, a sampling fault occurs on all-inone device5, and all-in-one device4 finish overwriting. If zones I, II and III overcurrent protections of b8 act and the overcurrent protections of b11, b12, b13 have not acted, by means of the proposed strategy in Fig. 6, the local action signal and trip command of b8 are shown in Fig. 12a. Current waveforms for lines that connected to faulty bus are shown in Fig. 12b.

Scenario 4: A ground fault occurs on L5 at $t=0.2 \mathrm{~s}$ is simulated. Meanwhile, a sampling fault occurs on all-inone device5, and all-in-one device4 finish overwriting. As shown in Fig. 13, zone I overcurrent protection of b8 did

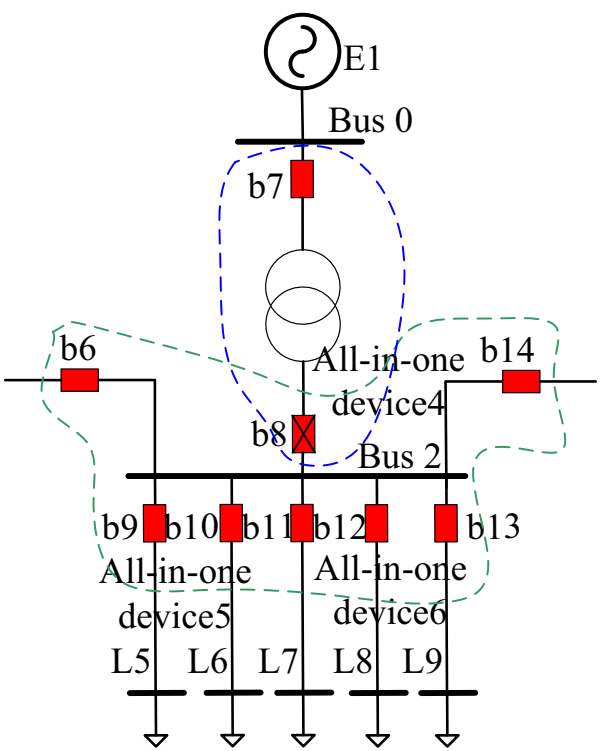

(a) Original differential loop

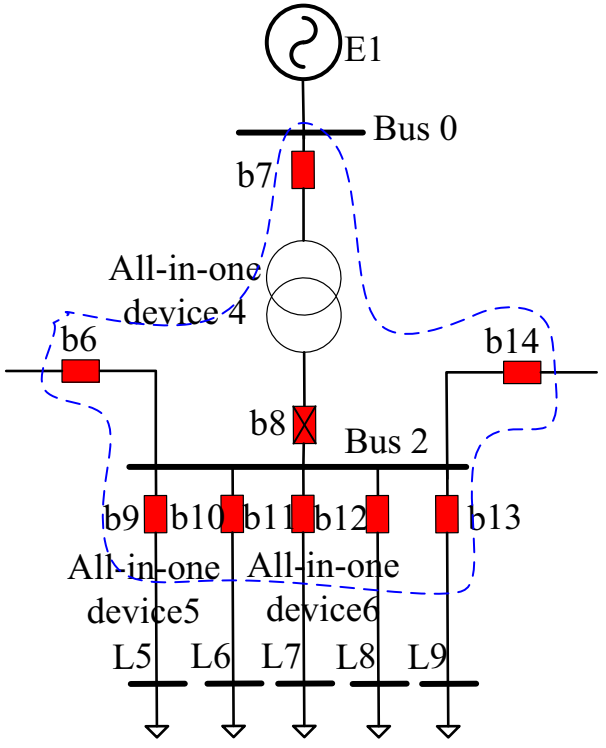

(b) Expanding differential loop

Fig. 15 Bus segment2 failure while b8 sampling value miss

not act and zones II and III act. In addition, b11, b12 and b13 have not detected overcurrent. On this occasion, b9 and b10 should be disconnected immediately.

Scenario 5: A ground fault occurs on L7 at $t=0.2 \mathrm{~s}$ is simulated. Meanwhile, a sampling fault occurs on all-inone device5, and all-in-one device4 finish overwriting. As seen in Fig. 14, zones I, II and III overcurrent protections of b8 acted. In addition, zones I, II and III of b11 also acted. Under this circumstance, b11 sends a block signal to $\mathrm{b} 8$, and segment I overcurrent protection of b8 sends a trip signal. 
Scenario 6: A ground fault occurs on Bus 2 at $t=0.2 \mathrm{~s}$ is simulated. Meanwhile, the sampling value of $b 8$ was missing, and devices finish overwriting. As seen in Fig. 15. The bus differential protection and transformer protection that consisted of b8 are blocked at first, then two small differential protection fuses to a large one. Obviously, the large differential protection could protect Bus 2 and the transformer.

\section{Conclusions}

This paper absorbs the advantage of hot backup in information field. Then, an all-in-one device based distributed substation area protection framework is proposed. Point-topoint model and VLAN based network-to network model are adopted in this framework. Dual redundancy on hardware and software level is realized by modularization, protection information mirroring storage and overwriting, which avoids the risk of single point failure of internal components effectively. On this basis, a distributed cooperative all-in-one device cluster based substation area joint defensive protection strategy is proposed. The proposed strategy can overcome the sampling, logic judgment and information transmission processes of protection may be mistaken by the atrocious conditions, and form a strong intelligent secondary device integrated network. EPOCHS based simulation examples verify the validity and rationality of the proposed architecture and strategy. If the secondary device is provided with adequate environmental reliability, the research of this paper will provide an excellent foundation for integration of primary and secondary device.

Acknowledgment This work was supported by the Science and Technology Project of State Grid Corporation of China (research and development of novel framework based protection adaptive to the evolution of substation and power grid).

Open Access This article is distributed under the terms of the Creative Commons Attribution 4.0 International License (http:// creativecommons.org/licenses/by/4.0/), which permits unrestricted use, distribution, and reproduction in any medium, provided you give appropriate credit to the original author(s) and the source, provide a link to the Creative Commons license, and indicate if changes were made.

\section{References}

[1] Babu S, Hilber P, Jürgensen JH (2014) On the status of reliability studies involving primary and secondary equipment applied to power system. In: Proceedings of the 2014 international conference on probabilistic methods applied to power systems (PMAPS'14), Durham, UK, 7-10 July 2014, 6 pp.
[2] Li JR, Song XC, Zhang XL et al (2013) Integration technology of primary and secondary equipment in smart substation. Electr Power Constr 34(6):16-21

[3] Bricker S, Gonen T, Rubin L (2001) Substation automation technologies and advantages. IEEE Comput Appl Power 14(3):31-37

[4] Ni YM, Yang Y, Fan C et al (2014) Discussion on integration of secondary devices in smart substations. Autom Electr Power Syst 38(3):194-199. doi:10.7500/AEPS20130226005

[5] Lei HT, Singh C, Sprintson A (2014) Reliability modeling and analysis of IEC 61850 based substation protection systems. IEEE Trans Smart Grid 5(5):2194-2202

[6] Chen LJ, Mei SW (2015) An integrated control and protection system for photovoltaic micro grids. CSEE J Power Energy Syst 1(1):36-41

[7] Ma J, Shi YX, Ma W et al (2015) Substation backup protection scheme based on multiple limitedly overlapped divisions. Electr Power Autom Equip 35(1):101-106

[8] Giovanini R, Hopkinson K, Coury DV (2006) A primary and backup cooperative protection system based on wide area agents. IEEE Trans Power Deliv 21(3):1222-1230

[9] Sheng S, Li KK, Chan WL et al (2010) Adaptive agent-based wide-area current differential protection system. IEEE Trans Ind Appl 46(5):2111-2117

[10] Li ZX, Yin XG, Zhang Z et al (2013) Wide-area protection fault identification algorithm based on multi-information fusion. IEEE Trans Power Deliv 28(3):1348-1355

[11] Liu Z, Chen Z, Sun HS et al (2015) Multiagent system-based wide-area protection and control scheme against cascading events. IEEE Trans Power Deliv 30(4):1651-1662

[12] Tong XY, Wang XR, Wang R et al (2013) The study of a regional decentralized peer-to-peer negotiation-based wide-area backup protection multi-agent system. IEEE Trans Smart Grid 4(2):1197-1206

[13] Heydari H, Abbasi V, Faghihi F (2009) Impact of switchinginduced electromagnetic interference on low-voltage cables in substations. IEEE Trans Electromagn C 51(4):937-944

[14] Lin XN, Tian Q (2006) Comparative analysis on current percentage differential protections using a novel reliability evaluation criterion. IEEE Trans Power Deliv 21(1):66-72

[15] Lin XN, Zhao F (2012) Universal wavefront positioning correction method on traveling-wave-based fault-location algorithms. IEEE Trans Power Deliv 27(3):1601-1610

[16] Lin XN, Zhao MH, Alymann K (2005) Novel design of a fast phase selector using correlation analysis. IEEE Trans Power Deliv 20(2):1283-1290

Fanrong WEI received the B.Sc. degree from Huazhong University of Science and Technology, Wuhan, China, in 2013. He is now the doctor candidate of Huazhong University of Science and Technology. His research interests include digital protection relaying and power system control

Mengqi YU received the B.Sc. degree from Hunan University, Changsha, China, in 2014. She is now the master candidate of Huazhong University of Science and Technology, Wuhan, China. Her research interests include digital protection relaying and power system control.

Zhiqian BO received his B.Sc. degree from Northeastern University, China in 1982 and Ph.D degree from The Queen's University of Belfast, UK in 1988. Presently, he is with AREVA T\&D Substation Automation Solution Business. 
Kun YU received the B.Sc. and M.S. degrees from Changsha University of Science and Technology, Changsha, China, in 2013. He is now the doctor candidate of Huazhong University of Science and Technology, Wuhan, China. His research interests include digital protection relaying and power system control.
Jingguang HUANG received the Ph.D degree in the school of electrical and electronic engineering from Huazhong University of Science and Technology (HUST), Wuhan, China. Currently, He is an associate professor of HUST. His research interests include modern signal processing and power system protective relaying. 